\title{
Rhetorice contracta (1621), II, 27, « Les discours de reproches et de condamnation sans appel »
}

Notes et édition du texte Francis Goyet, traduction Laurence Vianès

\section{G. J. Vossius}

Francis Goyet (éd.)

Traducteur : Laurence Vianès

\section{(2) OpenEdition Journals}

Édition électronique

URL : http://journals.openedition.org/rhetorique/190

DOI : $10.4000 /$ rhetorique.190

ISSN : 2270-6909

Éditeur

UGA Éditions/Université Grenoble Alpes

Édition imprimée

ISBN : 978-2-84310-263-9

Référence électronique

G. J. Vossius, «Rhetorice contracta (1621), II, 27, « Les discours de reproches et de condamnation sans appel » », Exercices de rhétorique [En ligne], 2 | 2013, mis en ligne le 18 novembre 2013, consulté le 12 septembre 2020. URL : http://journals.openedition.org/rhetorique/190 ; DOI : https://doi.org/10.4000/ rhetorique.190

Ce document a été généré automatiquement le 12 septembre 2020.

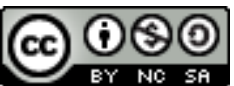

Les contenus de la revue Exercices de rhétorique sont mis à disposition selon les termes de la Licence Creative Commons Attribution - Pas d'Utilisation Commerciale - Partage dans les Mêmes Conditions 4.0 International. 


\section{Rhetorice contracta (1621), II, 27, "Les discours de reproches et de condamnation sans appel »}

Notes et édition du texte Francis Goyet, traduction Laurence Vianès

\section{G. J. Vossius}

Francis Goyet (éd.)

Traduction : Laurence Vianès

Texte de l'éd. de 1660 : Rhetorices contractae, sive Partitionum Oratoriarum libri quinque, Leipzig, Christian Kirchner, II, 27 (dernier chapitre), p. 221-223; $1^{\mathrm{e}}$ éd. Leyde, J. Maire, 1621 ; nombreuses rééd. jusqu'à 1698, dont certaines sur Google (qui a aussi l'éd. Madrid, Anton. Sancha, 1781). Rhetorices, en caractères latins, est le mot grec rhètorikè au génitif, litt. « les cinq livres de la Rhétorique abrégée ». Le titre Partitiones reprend celui de Cicéron, Partitiones Oratoriae, les Divisions de l'art oratoire : la rhétorique classée, pour les élèves.

Mêmes principes d'édition que précédemment.

Les références bibliographiques que nous mentionnons en abrégé dans les notes sont données dans la Bibliographie en fin du document précédent, Vossius, Institutiones (1605).

1 XXVII. Le discours de Reproches, de Condamnation sans appel et d'Appel à la clémence. DE EXPOSTULATIONE, EXPROBRATIONE ET DEPRECATIONE.

\section{$\S 1$ [Le discours de reproches]}

2 Le discours de reproches consiste à se plaindre d'une injustice qui nous a été faite.

$\S 1$. EXPOSTULATIO est querimonia de injuria accepta. 


\section{$\S 2$}

Deux cas se présentent : reproches légers, ou graves. Les légers doivent être tempérés soit par l'éloge, soit par la plaisanterie, soit par une habile façon de présenter les choses autrement [dissimulatio ${ }^{1}$ : si ce n'est peut-être lorsque nous sommes en autorité infiniment au-dessus de celui à qui nous nous adressons. Nous désapprouverons, certes, le fait commis : mais nous excuserons, autant que possible, celui qui l'a commis et n'a $\mathrm{pu}$ vouloir le commettre. Nous dirons donc: «Je m'étonne que tu aies négligé les devoirs de ta charge: mais je préfère tout imaginer plutôt que de penser que ce manquement puisse être dû chez toi à une diminution de l'amitié que tu as toujours portée ou à moi-même, ou à mes amis, ou à l'État. "

§ 2. Ea vel levior est, vel gravior. Levior, vel laude, vel joco, vel dissimulatione, temperandae est: nisi forte summae autoritatis apud alterum simus. Ac factum quidem improbabimus: excusabimus vero voluntatem, quantum licet. Dicemus, nos mirari, quod officium neglexerit: sed quidvis tamen malle suspicari, quam eo hoc factum esse, quia nostri, vel amicorum, vel Reip. amore minus tangatur, quam solet.

\section{§ 3}

Dans l'autre cas, celui des reproches graves, attention à ne pas les faire pour des raisons légères. Attention aussi, au moment de donner un comble d'importance au tort subi, à ne pas paraître en faire trop ${ }^{2}$ : que plutôt on croie que nous en avons supporté bien d'autres, sur lesquels jusqu'ici nous avions gardé le silence : mais que décidément le mal est allé si loin, qu'il n'est plus possible de le celer: d'autant que des maux plus grands sont même à craindre 3 .

§ 3. In graviori expostulatione videndum, ne ob leves causas fiat. et, ut gravis subsit causa, tamen ita est instituenda oratio, ut ne injuriam nimis videamur exaggerare: sed potius credamur multo plura tulisse, [p. 221] quae silentio hactenus presserimus: nunc autem eo processisse malum, ut dissimulari ultra non possit: praesertim cum majora etiam timeantur.

\section{[§ 3b]}

En effet, rien ne rend plus convaincante notre plainte, que si dans celle-ci brille notre patience à tout supporter. Car il s'en déduira que nous paraîtrons être contraints de nous plaindre sous le coup de la nécessité la plus impérieuse. C'est pourquoi il faut prendre peine, surtout si nous sommes un personnage haut placé [gravis], à ce que nos propos ne tiennent rien ni des gens du commun ni des femmes. Cela n'empêche pas que les affligés et les opprimés puissent recourir à un discours suscitant la pitié ${ }^{4}$, adressé à des supérieurs et <en général> à tous ceux de qui dépend leur salut. La façon de procéder sera différente, si nous nous adressons à des égaux, et que celui de qui nous nous plaignons, nous ne voulions pas le perdre, mais le ramener à de meilleures dispositions envers nous. Pour ce dernier cas, le modèle à suivre nous est ici donné par Cicéron lui-même, dans la première Philippique ${ }^{5}$ : "Mais avant de parler de la république, je me plaindrai en peu de mots de l'injure que $\mathrm{M}$. Antoine m'a faite hier. Je suis son ami ; et c'est un devoir pour moi de l'être, après le service qu'il m'a rendu; je l'ai toujours déclaré. [V.] Pourquoi donc me forcer si rudement hier à venir au sénat? étais-je seul absent? n'aviez-vous jamais été moins nombreux? l'affaire était-elle si grave que les malades eux-mêmes dussent s'y faire porter ? Hannibal était à nos portes, 
apparemment ; ou bien on délibérait sur la paix avec Pyrrhus : car, si nous en croyons l'histoire, c'est pour cela qu'Appius, vieux et aveugle, se fit porter au sénat. [12.] Il s'agissait d'actions de grâces: en pareil cas, les sénateurs n'ont pas coutume de manquer. Ce n'est pas à cause des gages, mais par intérêt pour ceux qu'on veut honorer. Il en est de même quand on délibère sur un triomphe. Les consuls y mettent si peu de soin, que les sénateurs sont, pour ainsi dire, libres de ne pas venir. Comme cet usage m'était connu, que j'étais fatigué du voyage, et que je me sentais mal disposé, j’ai cru qu'en considération de notre amitié, je pourrais me faire excuser auprès d'Antoine. Mais il a dit en votre présence qu'il viendrait avec des ouvriers pour abattre ma maison. C'était montrer beaucoup de colère et bien peu de modération. De quel crime étais-je donc coupable, pour qu'il ait osé dire qu'il renverserait avec des ouvriers de l'État une maison élevée aux frais de l'État, sur un décret rendu par vous? Qui a jamais fait de pareilles menaces à un sénateur pour le forcer de venir au sénat, et quelle peine y a-t-il au delà des gages et de l'amende? S'il avait su quel devait être mon avis, sans doute il eût été moins sévère. »

Nihil sane fidem magis adstruit querimoniae nostrae, quam si reluceat in ea animus patiens. Nam ita consequimur, ut videatur illam extorsisse necessitas. Itaque opera danda est, praesertim personae gravi; nequid abjectum ac muliebre habeat sermo. Attamen adflictis plane, atque oppressis, miserabili oratione uti licet apud superiores, et omnes, quorum in manu eorum sita est salus. Dispar vero ratio erit, si apud aequales dicamus; nec eum, de quo querimur, perditum ire velimus, sed ad mentem revocare saniorem. Eaque in re praeit nobis Tullius ipse Philip. I. «Priusquam, inquit, de Rep. dicere incipio, pauca querar de hesterna M. Antonii injuria, cui sum amicus: idque nonnullo ejus officio debere esse, prae me semper tuli. [Phil. I, V] Quid tandem erat causae, cur in senatum hesterno die acerbe cogerer? solusne aberam? an non saepe minus frequentes fuistis? an ea res agebatur, ut etiam agrotos deferri oporteret? Annibal, credo, erat ad portas, aut de Pyrrhi pace agebatur: ad quam causam etiam Apium illum et caecum, et senem, delatum esse, memoriae proditum est. [Phil. I, § 12] De supplicationibus referebatur: quo in genere senatores deesse non solent. Coguntur enim, non pignoribus, sed eorum, quorum de honore agitur, gratia; quod idem fit, cum de triumpho refertur. Ita sine cura consules sunt, [p. 222] ut pene liberum sit senatori non adesse. Qui cum mihi mos notus esset, cumque de via languerem, et mihimet displicerem: misi pro amicitia, qui hoc ediceret. At ille, vobis audientibus, cum fabris se domum meam venturum esse dixit. Nimis iracunde hoc quidem, et valde intemperanter. Cujus enim maleficii ista poena est, ut dicere in hoc ordine auderet, se publicis operis disturbaturum publice ex senatus sententia aedificatam domum? Quis autem unquam tanto damno senatorem coegit? aut quid est ultra pignus, aut multam? qui si scisset, quam sententiam dicturus essem, remisisset aliquid profecto de severitate cogendi. »

\section{$\S 4$ [Le discours de condamnation sans appel]}

6 S'ensuit le discours de condamnation sans appel, qui diffère du discours de reproches en ceci. Les reproches s'adressent à des amis, et la condamnation sans appel à des ennemis ; et encore, pas n'importe lesquels, mais seulement les ingrats ${ }^{6}$.

$\S 4$. Sequitur EXPROBRATIO. quae eo differt ab expostulatione, quod illa etiam fiat adversus amicos; exprobratio adversus inimicos; neque hos quosvis, sed ingratos.

\section{$\S 5$}

7 Ce type de discours a deux parties. La première consiste à rappeler le bienfait ${ }^{7}$ dont nous avons été l'auteur et même à lui donner un comble d'importance. Comme il peut 
paraître peu digne de notre libéralité que de mettre ainsi en avant nos bienfaits et de les porter aux nues, nous affirmerons hautement que nous ne le faisons que bien malgré nous, et contraints par les torts subis. Et ici on s'autorisera d'en appeler à la conscience $^{8}$ de notre adversaire.

§5. Constat partibus duabus. Prior est beneficii nostri commemoratio, et exaggeratio. Quia vero parum liberale videtur beneficia sua objectare, eaque extollere multum: eo affirmabimus, id nos facere invitos, sed alienis compelli injuriis. Atque hic et conscientiam adversarii testari licebit.

\section{$\S 6$}

8 La seconde partie consiste à exposer le méfait commis et en outre à en amplifier l'importance.

§ 6. Altera pars est expositio, nec non amplificatio maleficii alieni.

\section{$\S 7$}

Parfois aussi, on remonte de l'hypothèse à la thèse, et, sous forme générale, on dit deux mots sur ce défaut ou vice qu'est l'ingratitude.

Exemple ${ }^{9}$, la lettre de Cicéron à Antoine, $<$ Fam. $>\mathrm{V}, 5$. De même, cette lettre qui est un exercice d'école, ou l'œuvre de quelque professeur de rhétorique, signée Cicéron et adressée à Octave ${ }^{10}$. Et le discours d'Ariane à Thésée chez Catulle ${ }^{11}$, qui commence "Ainsi donc, perfide! tu ne m'as enlevée du palais d'un père ». Et le discours de Didon à Énée ${ }^{12}$, dont voici l'incipit, «Le barbare a-t-il été touché de mes pleurs? » De même, chez Ovide, la lettre de Médée à Jason ${ }^{13}$.

§. Quandoque et ab hypothesi ad thesin adscenditur, universeque aliquid dicitur de vitio criminis ingrati.

Exemplo sit epistola Ciceronis ad Antonium. [p. 223] quae quincta est libri quincti. item epistola Scholastici, seu rhetoris cujusdam, sub nomine Ciceronis ad Octavium. Ut et oratio Ariadnae ad Theseum apud Catullum ${ }^{14}$, cujus initium, Siccine me patriis abvectam perfide ab oris?

Et oratio Didus ad Aeneam ${ }^{15}$, quae incipit,

Nam fletu ingemuit nostro, etc.

Item apud Ovidium epist. Medeae ad Jasonem.

\section{NOTES}

1. présenter les choses autrement: sur ce sens de dissimulatio, voir la note 9 du $1^{\text {er }}$ document de l'Atelier (Vossius, 1605).

2. à ne pas paraître en faire trop : à ne pas paraître " exagérer " sous prétexte d'exaggeratio, donc à ne pas dépasser la juste mesure. L'exaggeratio en elle-même (voir note $22 \mathrm{du} 1^{\mathrm{er}}$ document) n'a pas le sens moderne d'exagération, elle consiste à porter à son comble une amplificatio. 
3. à craindre: c'est sans doute, par l'invocation du futur (le a futuro), une partie de menaces ou comminatio, pour susciter comme émotion la crainte, ou peut-être plutôt la terreur (timeantur; voir timor chez Pajot, fin de la note $22 \mathrm{du} 1^{\mathrm{er}}$ document).

4. un discours suscitant la pitié : rappel de ce que Junius nomme la lettre querula, plaintive. Voir querulus dans les notes 1 et $4 \mathrm{du} 1^{\mathrm{er}}$ document.

5. première Philippique : Phil. I, IV-V, 11-12 ; trad. Joseph-Victor Le Clerc (Paris, Werdet et Lequien fils, 1826-1827).

6. mais seulement les ingrats : $c f$. la première phrase d'Érasme, dans son chapitre «De exprobratione ", " Cum amicis expostulamus, ita inimicis et ingratis exprobramus » (De conscribendis..., chap. 61, LB I, 462 - pour les ouvrages ainsi cités, voir la Bibliographie du $1^{\mathrm{er}}$ document). Plus haut dans la Rhétorique abrégée (II, 26, §5), même distinguo, avec le même differt: l'objurgation "diffère (Differt) de l'invective, en ce que cette dernière en général vise des ennemis et que son but est de leur nuire, alors que la première s'adresse à des amis et s'efforce de leur être utile ». Vossius d'un traité à l'autre a évolué dans sa réflexion, en revenant à Érasme. Dans les Institutiones, il a rapproché l'exprobratio de l'objurgatio, donc a laissé entendre que même là l'orateur pouvait s'adresser à un " ami », ou tenter de maintenir l'alliance, tel Dieu dans l'Ancien Testament. Ici dans la Rhétorique abrégée, il durcit à nouveau les choses du côté de l'exprobratio, celle-ci ne saurait plus qu'être adressée à des "ennemis ». Dans ses deux traités, la réflexion de Vossius porte sur l'esprit même qui anime les paroles, sur ce que veut le locuteur (malimus, $1^{\text {er }}$ document, chap. XVII, $\S 1 \mathrm{~d}$ et $\S 2$ «néanmoins nous préférons conserver amitié et alliance »; velimus, $2^{\mathrm{e}}$ document, $\S 3$, « celui de qui nous nous plaignons, nous ne voulons pas le perdre »). Ce qui est déterminant pour qualifier un discours, c'est ce que vise l'auteur du discours, en latin l'intentio auctoris - le locuteur lui-même cherchant à sonder les intentions du destinataire (sa loyauté ou

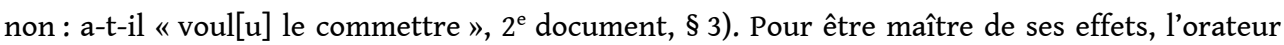
doit d'abord savoir si, dans sa tête, il s'adresse à un ami ou à un ennemi ; et une fois cette décision prise, choisir ses formules pour que son discours soit ressenti comme amical ou inimical, comme une déclaration de paix ou de guerre. Le véritable échec rhétorique serait par exemple de croire faire une répréhension « amicale », sans rupture de la relation, et d'être perçu comme faisant une invective « inamicale ", précipitant la rupture quand on voulait l'éviter. - Le retour à Érasme achève le détricotage par Vossius du chapitre de Junius sur l'expostulatio. Ce dernier fait passer le distinguo ami/ennemi entre les expostulationes « légère » et "grave » de Vossius : « vel cum amicis de neglecto officio : vel illata iniuria atque contumelia cum inimicis» (Scholae, p. 217). Du coup, Junius ne mentionne qu'au passage l'exprobratio, dont il ne fait même pas un chapitre à part. Vossius ne se contente pas de déplacer le curseur ami/ennemi : le critère essentiel selon lui n'est pas du côté de l'objet (la gravité ou non de l'accusation) mais du sujet (l'esprit dans lequel parle le locuteur).

7. rappeler le bienfait: renvoi au terme standard de commemoratio beneficiorum. Un peu après, «mettre en avant nos bienfaits » («beneficia... objectare ») : pour cette formule, voir la note $26 \mathrm{du}$ $1^{\text {er }}$ document.

8. en appeler à la conscience: ou plus exactement au témoignage («testari») de sa conscience, et pour ainsi dire à sa mauvaise conscience. La formule vient d'Érasme : «Conscientiam ipsius subinde testabimur » (dans le bref chap. 61 sur l'exprobratio du De conscribendis..., LB I, 462 ; suite du texte dans la note $22 \mathrm{du} 1^{\mathrm{er}}$ document). La conscientia est le juge intérieur, voir la note $1 \mathrm{du} 1^{\mathrm{er}}$ document (Ayliffe). Pajot fait de cet appel l'exorde ex abrupto de l'exprobratio (voir note $22 \mathrm{du} 1^{\mathrm{er}}$ document). Selon Melanchthon dans son commentaire à l'Énéide, la réplique de Didon à Énée est une exprobratio, et le tout début, «Tu n'es ni le fils d'une déesse » (IV, v. 365), est un « abruptum principium ». Melanchthon lui-même reprend cette dernière formule à Cristoforo Landino (1424-1504), dans le commentaire de celui-ci à Virgile (Publii Vergilii Bvcolica [...]. Ad Hos Donati Fragmenta, Christophori Landini..., Venise, 1507, pages non numérotées, ad locum; BSB00010391, image 00598, dernière ligne de la colonne de gauche). Landino commente le « ejectum » du v. 373 
par «beneficiorum exprobatio » et non exprobratio (image 00599, col. de droite). Sur la confusion entre exprobratio et exprobatio, voir note $21 \mathrm{du} 1^{\mathrm{er}}$ document.

9. Exemple: les exemples qui suivent illustrent l'ensemble des paragraphes sur l'exprobratio (§4-7), et pas seulement ce $\$ 7$. En effet, seul le discours d'Ariane chez Catulle a une généralisation (voir note $22 \mathrm{du} 1^{\mathrm{er}}$ document), à son début : le célèbre «Et maintenant, qu'aucune femme n'ajoute foi aux serments d'un homme », etc. (v. 143-148). - Vossius reprend à Érasme (voir note 6) trois de ses quatre exemples d'exprobratio : la Fam. V, 5 (Etsi statueram nullam) et la lettre à Octave, ainsi que Médée à Jason. Érasme cite aussi saint Jérôme à Julien (De conscribendis..., LB I, 463).

10. Octave: Pseudo-Cicéron, lettre à Octavianus, Si per tuas legiones mihi licitum fuisset.

11. Catulle: « Noces » ou «Épithalame de Thétis et de Pélée ». Catulle, Carmina, LXIV, v. 132-201; c'est Ariane qui parle, et ce sont ses « dernières plaintes » ou querelae : «extremis maestam dixisse querelis» (v. 130). Vossius cite ensuite le vers initial (v. 132), en mettant à la fin de celui-ci oris, variante que ne retiennent pas les éditions savantes actuelles, qui ont aris et traduisent « enlevée aux autels de mon père ». Pour rester au plus près du texte de Vossius, nous reprenons donc la trad. François Noël (Traduction complète des Poésies de Catulle, Paris, Arthus-Bertrand et Rémont, 1805, p. 145, Google), lequel a pour suite immédiate: «que pour me délaisser sur ce triste rivage!»

12. Didon à Énée : Énéide, IV, v. 369 (le discours est aux v. 365-387) ; trad. Desfontaines, 1743 (voir Francis Goyet, « La rhétorique de Didon dans l'acte II [de Didon se sacrifiant] : l'exprobratio au sein des vers 851-952 ", à paraître dans Lectures de Jodelle, éd. E. Buron et O. Halévy, Rennes, PUR). Cf. Ovide, Héroïdes, VII.

13. la lettre de Médée à Jason: Ovide, Héroïdes, XII, avec exprobrare au texte même, «Il y a quelque volupté à reprocher un bienfait à l'ingrat", "Est aliqua ingrato meritum exprobrare voluptas " (v. 21). À partir du v. 180, la lettre se clôt par les menaces, dont celle de tuer les enfants que Médée a eus de Jason : «Effroyables sont les menaces (minas) qu'enfante la colère » (v. 208). - Les Héroïdes, ou lettres fictives écrites par des héroïnes, nous donneront elles-mêmes une manière de clore, sur la différence entre expostulatio et exprobratio qui était notre sujet. De Médée à Didon, les héroïnes abandonnées recourent évidemment à l'exprobratio, violent reproche d'ingratitude, condamnation sans appel, avec lamentarsi ou plainte sur leurs malheurs: l'amant est un « ennemi », la relation est rompue. En revanche, quand Pénélope écrit à Ulysse qui depuis tant d'années erre en Méditerranée (Héroïdes, I), c'est une expostulatio (« Penelope expostulat cum Ulysse de diuturniore absentia. Ovid. in Epist. Heroidum ", Tesmar, Exercitationes..., VII, p. 619). Pénélope écrit comme si Ulysse pouvait encore revenir, dans sa tête la rupture ou divorce n'est pas encore consommé, malgré tous les torts qu'elle énumère. C'est, dans la première épître de Marot, la grande différence entre Maguelonne, qui attend, et Didon, qui se suicide : la « ferme amour » est une des figures de la foi ou alliance (fides ou amicitia) que l'on maintient en dépit de tout.

14. "Carmine de nuptiis Pelei et Thetidis. » (note de Vossius)

15. « Lib. 4. » (note de Vossius) 


\section{AUTEURS}

\section{G. J. vossius}

Gerhard Johann Voss, 1577-1649 